\title{
Impact of specialty of admitting physician and type of hospital on care and outcome for myocardial infarction in England and Wales during 2004-5: observational study
}

John Birkhead, Clive Weston, Derek Lowe, on behalf of the National Audit of Myocardial Infarction Project (MINAP)

Steering Group

\begin{abstract}
Objective To examine process of care and outcome for patients admitted with acute myocardial infarction to hospitals in England and Wales in relation to type of consultant care and type of hospital.

Design Observational study of 88782 patients admitted with myocardial infarction during 2004-5, using records from the national audit of myocardial infarction project (MINAP) database.

Outcome measures Use of reperfusion treatment and secondary prevention drugs, use of angiography, and 90 day mortality of patients admitted under the care of cardiologists and non-cardiologists in hospitals with and without facilities for coronary intervention.

Findings 36\% of patients were admitted under the care of a cardiologist and 20\% to a hospital with coronary interventional facilities. Patients admitted under cardiologists had fewer comorbidities than other patients and were more likely to have reperfusion treatment (12 266/14 $433(85 \%) v 13$ 682/17 064 $(80 \%))$ and appropriate secondary prevention drugs. Overall, $27431 / 79374$ (35\%) of patients had angiography. Relatively more patients admitted to interventional hospitals

$(8167 / 14661 ; 56 \%)$ than to other hospitals had angiography (19 264/64 713; 30\%). The adjusted risk of death by 90 days for patients treated in interventional compared with non-interventional hospitals was 0.93 (95\% confidence interval 0.82 to 1.06$)$. The adjusted risk of death at 90 days for patients admitted under cardiologists compared with non-cardiologists was 0.86 (0.81 to 0.91$)$.

Conclusions Patients cared for by cardiologists had less comorbidity than other patients. They were more likely to receive proved treatments and angiography, and they had a lower adjusted 90 day mortality. Large differences existed in the use of angiography between interventional and non-interventional hospitals. These findings show wide variations in the management and outcome of patients with myocardial infarction in England and Wales.
\end{abstract}

\section{Introduction}

The United Kingdom has few cardiologists per capita. In 2000, the latest available comparative data, there were 12 cardiologists per million population, the second lowest of all European countries. ${ }^{1}$ A patient admitted to hospital with acute myocardial infarction may therefore not come under the care of a cardiolo- gist. The subsequent involvement of a cardiologist is not routine, and some patients with myocardial infarction have no contact with a cardiologist throughout their hospital stay or at follow-up after discharge. ${ }^{2}$ Treatment options for the care of acute coronary ischaemic syndromes have become increasingly complex. We therefore questioned whether management of acute myocardial infarction provided by cardiologists and non-cardiologists had the same outcomes.

We examined 88782 records from the national audit of myocardial infarction project (MINAP) database entered between 1 January 2004 and 31 March 31 2005, to compare treatment, use of investigations, and 90 day mortality between patients with acute myocardial infarction who were admitted under the immediate care of cardiologists and patients admitted under non-cardiologists.

\section{Methods}

The development and early findings of MINAP have been described elsewhere. ${ }^{34}$ The project uses a dataset that allows examination of pre-hospital and in-hospital care of all acute coronary syndromes and is a part of the NHS data dictionary. ${ }^{5}$ The project is based on the technological platform developed by the Central Cardiac Audit Database Group. ${ }^{6}$ The primary purpose of the project is to provide hospitals with contemporary online analyses of their individual performance and comparisons with national aggregate data.

We analysed records for patients admitted between 1 January 2004 and 31 March 2005 who received a final diagnosis of myocardial infarction. Patients subsequently transferred from the admitting hospital to a hospital with facilities for coronary intervention for further treatment were only counted once. We examined mortality outcome at 90 days for patients who had valid NHS numbers linking their admission with the Office of National Statistics.

The term "care under a cardiologist" indicates that the patient was admitted under the direct responsibility of a cardiologist and received care from a cardiologist and his or her team during at least the first 24 hours of the admission. Where care was initially under a non-cardiologist, we could not establish the subsequent involvement of a cardiologist.

Of 230 hospitals in England and Wales, 41 had interventional facilities on site at March 2004. These ranged from hospitals treating only their own patients to large institutions doing high volumes of interventions for emergency patients from neighbouring hospitals. 


\section{Statistical analysis}

We present patients' data as percentages and ratios of percentages and inter-hospital variation statistics as medians and interquartile ranges. We used binary regression methods (Stata 8 "binreg" software) to assess 90 day mortality and process measures (reperfusion treatment, angiography, and secondary prevention drugs) to obtain risk ratios adjusted for hospital clustering and covariates. We analysed the "absence" rather than the "presence" of some treatments to minimise constraints on risk ratio values. The covariates comprised age $(<55,55-64,65-74$, $75-84$, and $\geq 85$ years), sex, smoking status, type of infarction (presence or absence of ST elevation), and 10 comorbid conditions-previous infarction, previous angina, treated hypertension, treated hyperlipidaemia, treated heart failure, diabetes, peripheral vascular disease, cerebrovascular disease, chronic renal failure with creatinine $>200 \mathrm{mmol}$, and chronic obstructive pulmonary disease or asthma. We condensed comorbidity status into simple counts of conditions to enable the iterative processes in the regression analyses to converge. We took care not to adjust for factors that could lie on the causal pathway between the admitting consultant and the dependent variable. ${ }^{7}$ We coded missing data for categorical variables as a response category to maintain patient denominators.

\section{Results}

We analysed data on 88782 patients admitted to hospital with a diagnosis of myocardial infarction at death or discharge. Of these, 83599 were admitted under a cardiologist or a physician whose specialty interest was not cardiology (non-cardiologist), and $80 \%$ were admitted to hospitals without facilities for coronary intervention (figure). Of 230 hospitals, 41 (18\%) had interventional facilities, with $57 \%$ of patients admitted under a cardiologist (hospital median 69\%, interquartile range 27-95\%). In 189 non-interventional hospitals, $31 \%$ of patients were admitted under a cardiologist (hospital median 19\%, 9-41\%). Overall, $30383(36 \%)$ patients were admitted under a cardiologist.

\section{Patient characteristics}

Patients admitted under a cardiologist were younger, with a median age of 69 (interquartile range 58-78) years, compared with 73 (62-82) years for those admitted under a noncardiologist, and were more likely to be male, to be current smokers, to have electrocardiographic appearances of ST segment elevation, and to have lower comorbidity (table 1). After stratification by age, lower rates of heart failure, diabetes, cerebrovascular disease, chronic obstructive pulmonary disease, and chronic renal failure (group 1 comorbidities) persisted for patients admitted under cardiologists. We found similar rates for the other comorbid conditions listed in table 1 (group 2 comorbidities). We took simple counts of group 1 ( $0,1,2$ or more) and group $2(0,1,2,3$ or more) comorbidities as covariates in later regression analyses. Of all infarctions, 33 163/83 599 (40\%) showed ST segment elevation; cardiologists cared for 15282 $(46 \%)$ of these patients. By contrast, non-cardiologists cared for $35335(70 \%)$ of all non-ST elevation infarctions. The association between admitting consultant and type of infarction persisted after age stratification.

\section{Reperfusion treatment for ST elevation infarction}

Of 33163 patients with ST segment elevation infarction, details of reperfusion treatment were recorded for 33063 (99.7\%). Of these, 1566 had thrombolytic treatment before arrival in hospital and were not included in analyses of reperfusion treatment. Those admitted under a cardiologist were more likely to receive reperfusion treatment-12 266/14 433 (85\%), including primary angioplasty for 1310 (9.1\%), compared with $13682 / 17064$ $(80 \%)$ under a non-cardiologist, of whom $107(0.6 \%)$ had primary angioplasty. Overall, reperfusion treatment was used to a similar degree in non-interventional hospitals-20 413/24 686 $(83 \%)$ against 5535/6811 (81\%) in interventional hospitals. The risk of not receiving reperfusion treatment increased with age, but this risk was lower in each age band for patients admitted under a cardiologist (table 2). In both interventional and non-interventional hospitals, the adjusted risk was lower for patients cared for by a cardiologist -0.63 (95\% confidence interval 0.45 to 0.87 ) and 0.84 ( 0.74 to 0.96 ).

\section{Secondary prevention drugs}

We examined the use of aspirin, $\beta$ blockers, statins, and angiotensin converting enzyme inhibitors for 57508 patients who were discharged from the admitting hospital (we excluded inpatient deaths, transfers to other hospitals for further investigation, and unknown destinations). Patients in whom treatment was considered to be contraindicated or not indicated or was not given were analysed together. The proportion not receiving secondary prevention drugs increased with age, but in each age band this was lower for patients admitted under a cardiologist (table 3). The adjusted ratios for non-use of these drugs after admission under a cardiologist relative to a noncardiologist were aspirin 1.00 (95\% confidence interval 0.86 to 1.15), $\beta$ blockers 0.92 ( 0.87 to 0.97 ), statins 0.83 ( 0.71 to 0.97 ), and angiotensin converting enzyme inhibitors 0.98 ( 0.91 to 1.06$)$.

\section{Use of angiography}

Information on use of angiography was available for 79374 $(95 \%)$ patients. Angiography, including that done as part of the

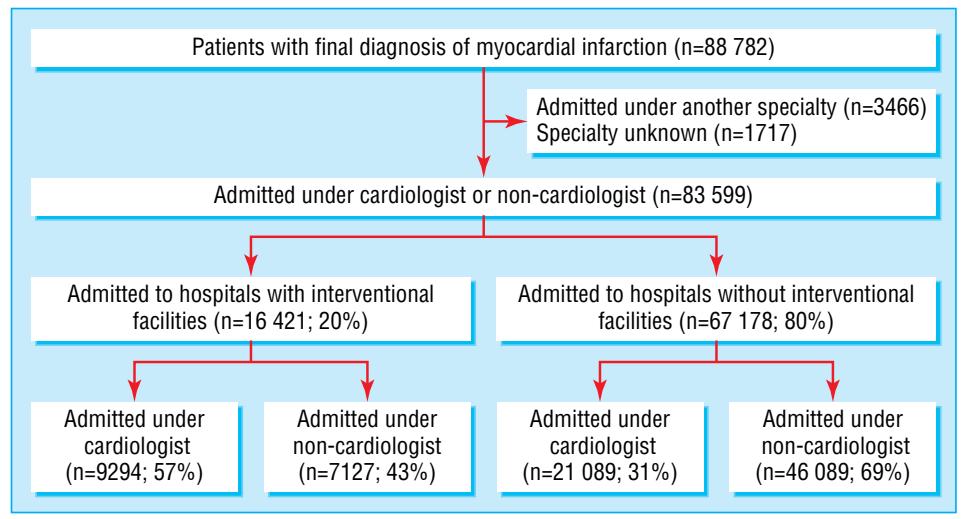

Patient cohort. 
Table 1 Patient characteristics. Values are numbers (percentages) unless stated otherwise

\begin{tabular}{|c|c|c|c|c|}
\hline \multirow{3}{*}{ Characteristic } & \multicolumn{2}{|c|}{ Cardiologist (n=30 383) } & \multicolumn{2}{|c|}{ Non-cardiologist ( $\mathrm{n}=53216$ ) } \\
\hline & & Inter-hospital median (IQR) & & Inter-hospital median (IQR) \\
\hline & All patients & $\%$ & All patients & $\%$ \\
\hline Age $>75$ years & $9611 / 30080(32.0)$ & $31(23-38)$ & 23 668/52 140 (45.4) & $43(35-51)$ \\
\hline Male & 20 538/30 252 (67.9) & $69(65-74)$ & 33 256/53 065 (62.7) & $64(59-68)$ \\
\hline Current smoker & $9442 / 27538$ (34.3) & $33(26-40)$ & 12 985/47 267 (27.5) & $28(23-33)$ \\
\hline ST elevation MI & 15 282/30 383 (50.3) & $50(39-68)$ & $17881 / 53216$ (33.6) & $34(25-51)$ \\
\hline \multicolumn{5}{|l|}{ Comorbidity* } \\
\hline \multicolumn{5}{|l|}{ Group 1: } \\
\hline Heart failure & $1229 / 25156(4.9)$ & $3(1-7)$ & $3900 / 48580(8.0)$ & $6(3-11)$ \\
\hline Diabetes & $5033 / 28076(17.9)$ & $17(13-22)$ & 10 109/49 079 (20.6) & $20(16-25)$ \\
\hline Cerebrovascular disease & 1863/25 $632(7.3)$ & $6(3-9)$ & $4698 / 48270(9.7)$ & $9(6-12)$ \\
\hline Obstructive pulmonary disease & $3317 / 25278(13.1)$ & $13(9-16)$ & $7567 / 48584$ (15.6) & $15(12-19)$ \\
\hline Chronic renal failure & $729 / 25144(2.9)$ & $2(0-4)$ & $2011 / 48578(4.1)$ & $3(1-5)$ \\
\hline One or more of above & $9673(31.8)$ & 33 (26-39) & $21206(39.8)$ & $40(31-47)$ \\
\hline \multicolumn{5}{|l|}{ Group 2: } \\
\hline Previous acute MI & 7059/29005 (24.3) & $24(17-29)$ & $13644 / 50743(26.9)$ & $26(21-31)$ \\
\hline Previous angina & $8618 / 28707(30.0)$ & $29(20-35)$ & $16700 / 49887$ (33.5) & 33 (26-38) \\
\hline Treated hyperlipidaemia & 7885/26913 (29.3) & $28(18-40)$ & $12872 / 47671(27.0)$ & $25(18-38)$ \\
\hline Treated hypertension & $12534 / 28693$ (43.7) & $44(37-50)$ & 22 741/49 938 (45.5) & $45(38-51)$ \\
\hline Peripheral vascular disease & $1357 / 27130(5.0)$ & $4(1-6)$ & $2620 / 48508(5.4)$ & $5(2-7)$ \\
\hline One or more of above & $11044(36.3)$ & $35(26-43)$ & $20502(38.5)$ & $38(29-44)$ \\
\hline
\end{tabular}

IQR=interquartile range; $\mathrm{MI}=$ myocardial infarction.

*Divided into two groups: in group 1, differences in frequency persisted after age stratification; in group 2, differences did not persist.

initial reperfusion strategy, was done for $27431(35 \%)$ patients. It was done more often in interventional hospitals $(56 \% v 30 \%)$ and for patients admitted under cardiologists (44\% v 30\%). Use of angiography was strongly associated with age (table 4). In non-interventional hospitals, the overall adjusted risk ratio for having angiography after admission under a cardiologist relative to a non-cardiologist was 1.20 (1.07 to 1.38$)$; in interventional hospitals, the adjusted ratio was 1.10 (0.97 to1.25).

Table 2 Non-use of reperfusion treatment (hospital thrombolytic treatment or primary angioplasty) for patients with ST elevation infarction ( $\mathrm{n}=31$ 497). Values are numbers (percentages) unless stated otherwise

\begin{tabular}{|c|c|c|c|c|c|c|}
\hline & \multicolumn{3}{|c|}{ Interventional hospitals } & \multicolumn{3}{|c|}{ Non-interventional hospitals } \\
\hline & Cardiologist & Non-cardiologist & Risk ratio* & Cardiologist & Non-cardiologist & Risk ratio* \\
\hline \multicolumn{7}{|l|}{ Age (years): } \\
\hline$<55$ & 108/1156 (9.3) & $61 / 353$ (17.3) & 0.54 & 198/2064 (9.6) & 330/2768 (11.9) & 0.80 \\
\hline 55 to 64 & 138/1145 (12.1) & 63/365 (17.3) & 0.70 & $240 / 2281(10.5)$ & $374 / 3319(11.3)$ & 0.93 \\
\hline 65 to 74 & $177 / 1201$ (14.7) & $116 / 482(24.1)$ & 0.61 & $390 / 2555(15.3)$ & 625/3706 (16.9) & 0.91 \\
\hline 75 to 84 & $225 / 1040(21.6)$ & 171/477 (35.8) & 0.60 & 412/1996 (20.6) & $956 / 3711(25.8)$ & 0.80 \\
\hline$\geq 85$ & $98 / 308(31.8)$ & $105 / 213$ (49.3) & 0.65 & $158 / 558$ (28.3) & $503 / 1289(39.0)$ & 0.73 \\
\hline Age missing & $9 / 41(22.0)$ & $5 / 30(16.7)$ & 1.32 & $14 / 88(15.9)$ & $73 / 351 \quad(20.8)$ & 0.76 \\
\hline Total & $755 / 4891$ (15.4) & $521 / 1920(27.1)$ & 0.57 & 1412/9542 (14.8) & 2861/15 144 (18.9) & 0.78 \\
\hline Inter-hospital median (IQR) & $11(4-19)$ & $25(4-50)$ & & $13(4-21)$ & $18(10-27)$ & \\
\hline
\end{tabular}

IQR=interquartile range.

${ }^{*}$ For reperfusion treatment not being used for patients admitted under a cardiologist relative to patients admitted under a non-cardiologist.

Table 3 Proportions of patients who did not receive secondary prevention drugs*

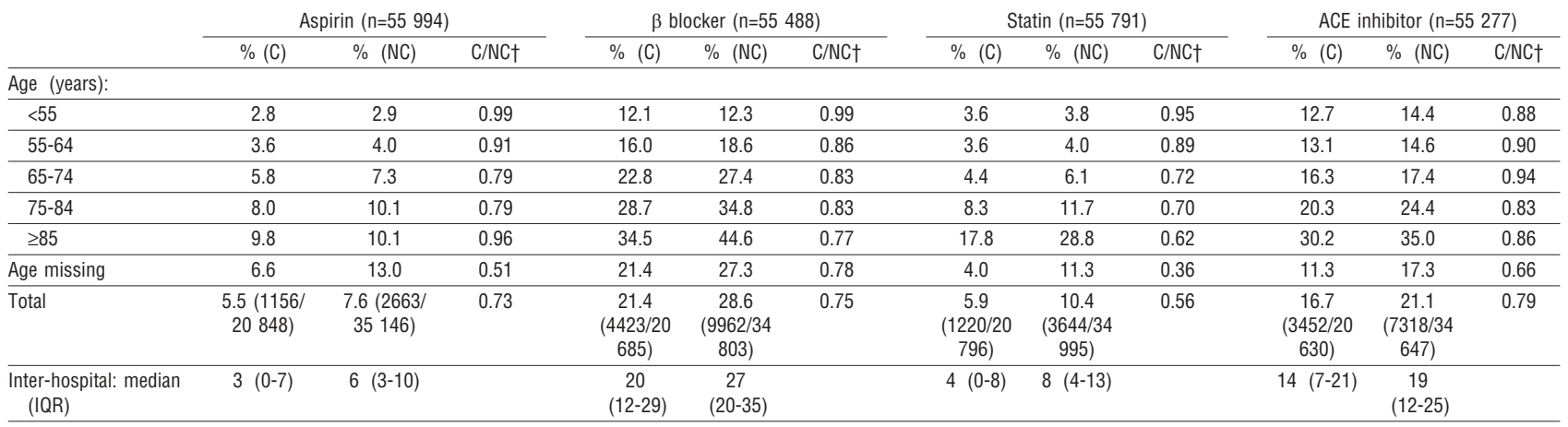

$\mathrm{ACE}=$ angiotensin converting enzyme; IQR=interquartile range.

*Patients for whom treatment was considered to be contraindicated or not indicated or was not given are grouped together. Patients who died in hospital, who were transferred to another hospital, or whose discharge destination was unknown are excluded.

†Risk ratio for secondary prevention treatment not being used for patients admitted under a cardiologist (C) relative to patients admitted under a non-cardiologist (NC). 
Table 4 Use of angiography ( $\mathrm{n}=79$ 374). Values are numbers (percentages) unless stated otherwise

\begin{tabular}{|c|c|c|c|c|c|c|}
\hline & \multicolumn{3}{|c|}{ Interventional hospitals } & \multicolumn{3}{|c|}{ Non-interventional hospitals } \\
\hline & Cardiologist & Non-cardiologist & Risk ratio* & Cardiologist & Non-cardiologist & Risk ratio* \\
\hline \multicolumn{7}{|l|}{ Age (years): } \\
\hline$<55$ & $1445 / 1773(81.5)$ & $600 / 747(80.3)$ & 1.01 & $1954 / 3668$ (53.3) & $2750 / 5765(47.7)$ & 1.12 \\
\hline 55 to 64 & 1428/1854 (77.0) & $740 / 1022(72.4)$ & 1.06 & 2037/4384 (46.4) & $3140 / 7615$ (41.2) & 1.13 \\
\hline 65 to 74 & $1484 / 2214(67.0)$ & $868 / 1506(57.6)$ & 1.16 & 2225/5581 (39.9) & $3461 / 10578(32.7)$ & 1.22 \\
\hline 75 to 84 & $845 / 2040(41.4)$ & $554 / 1865$ (29.7) & 1.39 & 1085/4898 (22.2) & 1992/13 397 (14.9) & 1.49 \\
\hline$\geq 85$ & $73 / 555$ (13.2) & $57 / 955(6.0)$ & 2.20 & $85 / 1500(5.7)$ & $225 / 6114$ (3.7) & 1.54 \\
\hline Age missing & $33 / 56(58.9)$ & $40 / 74(54.1)$ & 1.09 & $83 / 231$ (35.9) & $227 / 982(23.1)$ & 1.55 \\
\hline Total & $5308 / 8492$ (62.5) & $2859 / 6169$ (46.3) & 1.35 & $7469 / 20262(36.9)$ & $11795 / 44451$ (26.5) & 1.39 \\
\hline Inter-hospital: median (IQR) & $73(52-85)$ & $51(26-68)$ & & $31(17-53)$ & $23(12-40)$ & \\
\hline
\end{tabular}

$\mathrm{IQR}=$ interquartile range.

* Risk ratio of angiography being done for patients admitted under a cardiologist relative to those admitted under a non-cardiologist.

\section{Outcome}

All cause mortality at 90 days was $14.0 \%$ among $76376(91 \%)$ patients with a valid NHS number. Of patients with ST elevation infarction, 3511/29 $814(11.8 \%)$ died; of those with non-ST elevation infarction, 7170/46562 (15.4\%) died. All cause mortality was strongly associated with age. It was lower for patients admitted under cardiologists, both for those with ST elevation and non-ST elevation infarction and in both interventional and non-interventional hospitals (table 5). The adjusted risk ratio for mortality in interventional hospitals relative to noninterventional hospitals was 0.93 (0.82 to 1.06). Adjusted 90 day mortality risk was lower for patients admitted under a cardiologist for both ST elevation and non-ST elevation infarctions in both interventional and non-interventional hospitals (table 6).

\section{Discussion}

In English and Welsh hospitals, most patients who had acute myocardial infarction were admitted under the care of non-cardiologists, and the great majority were admitted to hospitals without facilities for coronary intervention. Patients not admitted under a cardiologist had a substantially higher all cause mortality during the 90 days after admission in both types of hospital setting and after adjustment for case mix.

\section{Patient characteristics and selection bias}

For most patients who present with myocardial infarction, immediate care, including provision of thrombolytic treatment, is provided by clinicians in emergency departments. ${ }^{4}$ Subsequent arrangements for care of patients with myocardial infarction vary widely between hospitals. In 2000 care for myocardial infarction was provided throughout the admission by the physi- cian who admitted the patient in 50\% of English hospitals, whereas routine transfer to a cardiologist occurred in only $23 \%{ }^{2}$ Although these figures may have changed with the appointment of more cardiologists, care for most patients is still provided by non-cardiologists. These data do not reveal the degree of cardiological input into the care of patients admitted under non-cardiologists, but when care was reported initially to have been under a non-cardiologist the 90 day mortality outcome was poorer than when care was provided by a cardiologist.

Within this varying pattern of care, cardiologists either select, or have referred to them, a population of patients who are younger, and thus more likely to be male and to have fewer comorbid conditions, and who are more likely to have ST elevation infarction, which has a lower 90 day mortality than non-ST elevation infarction. By contrast, non-cardiologists cared for $70 \%$ of patients with non-ST elevation infarction.

These differences reflect a selection bias, which may explain some of the differences in crude mortality data. The apparent "cherry picking" of patients at lower risk and with less comorbidity is not limited to British cardiologists. ${ }^{8}$ However, where differences in mortality have previously been shown between patients managed by cardiologists and by generalists, these were considerably attenuated when adjusted for age and comorbidity, a finding confirmed by our work. ${ }^{9}$

These data do not explain the mechanism by which selection bias occurs. We speculate that an inaccurate perception that non-ST segment elevation infarction carries a lower risk of mortality than ST segment elevation infarction might explain the increased likelihood of patients with non-ST segment elevation myocardial infarction being referred to non-cardiologists. Similarly, differences in outcome might reflect referral of older

Table 590 day all cause mortality for patients admitted under cardiologists and non-cardiologists and in interventional and non-interventional hospitals $(n=76376)$. Values are numbers (percentages) unless stated otherwise

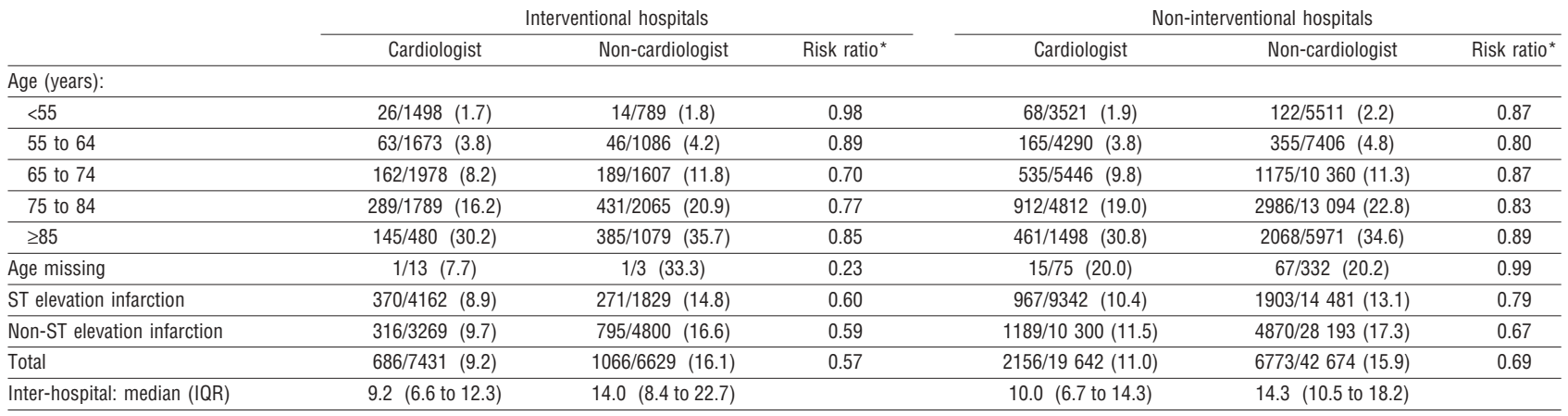


Table 6 Adjusted 90 day mortality risk (95\% confidence interval) for patients admitted under cardiologists relative to non-cardiologists for ST elevation and non-ST elevation infarction in interventional and non-interventional hospitals

\begin{tabular}{lccc} 
& Interventional hospitals & $\begin{array}{c}\text { Non-interventional } \\
\text { hospitals }\end{array}$ & All hospitals \\
\hline $\begin{array}{l}\text { ST elevation } \\
\text { infarction }\end{array}$ & $0.76(0.64$ to 0.90$)$ & 0.91 (0.84 to 0.98$)$ & 0.88 (0.82 to 0.95) \\
\hline $\begin{array}{l}\text { Non-ST elevation } \\
\text { infarction }\end{array}$ & $0.82(0.69$ to 0.98$)$ & 0.85 (0.78 to 0.92$)$ & 0.84 (0.78 to 0.91$)$ \\
\hline All infarctions & $0.81(0.69$ to 0.94$)$ & $0.87(0.82$ to 0.93$)$ & 0.86 (0.81 to 0.91$)$ \\
\hline
\end{tabular}

patients with greater comorbidity to non-cardiologists. However, this is not supported by any clear trend towards increasing risk ratios of death in higher age bands

\section{Treatment}

Patients admitted under cardiologists were more likely to receive reperfusion treatment for ST elevation infarction than those who were not, and they were more likely to have primary angioplasty. However, the number who had primary angioplasty in this study $(4 \%)$ was too small to have had any impact on differences in outcome. Cardiologists used secondary prevention drugs more intensively, although these differences were attenuated after adjustment. The effect of these treatment differences is unlikely to account for the differences in mortality. Other studies have confirmed that although cardiologists are more likely to prescribe drugs that may improve outcome, the use of these drugs has not always been associated with lower mortality after adjustment for patients' and hospitals' characteristics. ${ }^{10-12}$

\section{Angiography}

Randomised trials of early revascularisation after myocardial infarction have shown benefit in terms of morbidity and mortality, and national and international societies now recommend the early use of diagnostic angiography after acute cardiac ischaemic events. ${ }^{13-19}$ Despite these recommendations, we found that only $18 \%$ of hospitals offered interventional facilities and that the overall rate of angiography (35\%) was low compared with other regions of the world. ${ }^{20}$ Although patients admitted to a hospital with interventional facilities were a minority, they were almost twice as likely to have angiography as those who were admitted to other hospitals. However, we found no significant difference in adjusted mortality by 90 days between patients who were treated in hospitals with interventional facilities and those who were not, a finding consistent with a recent registry study. ${ }^{20}$

\section{Comment}

The relatively small differences in practice between cardiologists and non-cardiologists, and apparent selection bias, do not explain the differences in mortality outcome described here. We speculate that other management differences must exist, which have an impact on mortality. In the past decade, treatment options for patients of all ages with myocardial infarction have become more complex, and ideally cardiologists and their teams should be closely involved in their care. These findings do not support the view that care for myocardial infarction should remain within the remit of the non-cardiologist. In a healthcare system committed to equity of access and outcome, the differences described here should raise questions about the model of care for patients with myocardial infarction in England and Wales. ${ }^{21}$

We acknowledge the contribution of the MINAP team; Lynne Walker (project manager), Felicity Naughton, and Anna-Maria Bunn at the Clinical Effectiveness and Evaluation Unit, Royal College of Physicians, London; and the project director of the Central Cardiac Audit Database, David Cunningham.

Contributors: JB conceived the work, did the initial analysis of the database, and wrote the first draft of the manuscript. CW read, criticised, and developed the text. DL corrected and refined the statistical analyses and produced the final version of the tables. Each author edited the final version and has approved the final text. JB is the guarantor.

Funding: The national audit of myocardial infarction is funded by the Healthcare Commission.

Competing interests: None declared.

Ethical approval: Not needed.

1 Block P, Weber H, Kearney P, Cardiology Section of the European Union of Medical Specialists. Manpower in cardiology II in western and central Europe (1999-2000). Eur Heart J 2003;24:299-310.

2 Birkhead JS, Georgiou A, Knight L, Walker L, Lowe D, eds. A baseline survey of facilities for the management of acute myocardial infarction in England 2000. London: Royal College of Physicians, 2001.

3 Birkhead JS, Pearson M, Norris RM, Rickards AF, Georgiou A. The national audit of myocardial infarction: a new development in the audit process. Journal of Clinical Excellence 2002:4:379-85.

4 Birkhead JS, Walker L, Pearson M, Weston CM, Cunningham AD, Rickards AF. Improving care for patients with acute coronary syndromes: initial results from the national audit of myocardial infarction project (MINAP). Heart 2004;90:1004-9.

5 Royal College of Physicians. MINAP data set. www.rcplondon.ac.uk/college/ceeu/ ceeu_ami_home.htm (accessed 24 Dec 2005).

6 Rickards $\bar{A}$, Cunningham D. From quantity to quality: the central cardiac audit database project. Heart 2000;82:18-22.

7 Leon DA. Failed or misleading adjustment for confounding. Lancet 1993;342:479-81.

8 Ayanian JZ, Guadagnoli E, McNeil BJ, Cleary PD. Treatment and outcomes of acute myocardial infarction among patients of cardiologists and generalist physicians. Arch Intern Med 1997;157:2570-6.

9 Chen J, Radford MJ, Wang Y, Krumholz HM. Care and outcome of elderly patients with acute myocardial infarction by physician specialty: the effects of co-morbidity and acute myocardial infarction by physician specialty

10 Go AS, Rao RK, Dauterman KW, Massie BM. A systematic review of the effects of physician specialty on the treatment of coronary disease and heart failure in the United States. Am J Med 2000;108:259-61

11 Frances CD, Go AS, Dauterman KW, Deosaransingh K, Jung DL, Gettner S, et al. Outcome following acute myocardial infarction: are differences among physician specialties the result of quality of care or case mix? Arch Intern Med 1999;159:1429-36.

12 Jollis JG, DeLong ER, Peterson ED, Muhlbaier LH, Fortin DF, Califf RM, et al. Outcome of acute myocardial infarction according to specialty of the admitting physician. $N$ Engl JMed 1996;335:1880-7.

13 Bach RG, Cannon CP, Weintraub WS, DiBattiste P, Demopoulos LA, Anderson HV, et al. The effect of routine, early invasive management on outcome for elderly patients with non ST segment elevation acute coronary syndrome. Ann Intern Med with non ST segm

14 Fox KA, Poole-Wilson PA, Henderson, Clayton TC, Chamberlain DA, Shaw TR, et al. Interventional versus conservative treatment for patients with unstable angina of non

\section{What is already known on this topic}

Different outcomes and treatment patterns have been shown for care of myocardial infarction by cardiologists and non-cardiologists in the United States

Whether similar differences exist in England and Wales is not known

Whether differences in care and outcome exist between patients admitted to hospitals with and without facilities for coronary intervention is not known

\section{What this study adds}

The third of patients with a myocardial infarction admitted under the care of a cardiologist were more likely to have reperfusion treatment and secondary prevention drugs

Patients admitted under a cardiologist were more likely to have angiography in hospitals both with and without interventional facilities

The adjusted 90 day mortality did not differ between interventional and non-interventional hospitals but was significantly lower for patients cared for by cardiologists than by non-cardiologists 


\section{Research}

ST elevation myocardial infarction: the British Heart Foundation RITA 3 randomised trial. Lancet 2002;360:743-51.

15 Kaul P, Armstrong PW, Chang W-C, Naylor CD, Granger CB, Lee KL, et al. Long-term mortality of patients with acute myocardial infarction in the United States and Canada: comparison of patients enrolled in global utilisation of streptokinase and t-pa for occluded coronary arteries (GUSTO-1). Circulation 2004;110:1754-60.

16 Van de Werf F, Ardissino D, Betriu A, Cokkinos D, Falk E, Fox KA, et al. Management of acute myocardial infarction in patients presenting with ST-segment elevation. Eur Heart 2003;24:28-66

17 Bertrand ME, Simoons ML, Fox KAA, Wallentin LC, Hamm CW, McFadden E, et al Management of acute coronary syndromes in patients presenting without persistent ST seoment eovation, Eur Heart $2002 \cdot 23: 1809-40$.

18 Braunwald E, Antman EM, Beasley JW, Califf RM, Cheitlin MD, Hochman JS, et al ACC/AHA 2002 guideline update for the management of patients with unstable angina and non-ST-segment elevation myocardial infarction: a report of the American College of Cardiology/American Heart Association Task Force on Practice Guideline (Committee on the Management of Patients With Unstable Angina). www.acc.org/ clinical/guidelines/unstable/incorporated/index.htm (accessed 24 Dec 2005).

19 British Cardiac Society Guidelines and Medical Practice Committee, Royal College of Physicians Clinical Effectiveness and Evaluation Unit. Guideline for the management of patients with acute coronary syndromes without persistent ECG ST segment elevation. Heart 2001;85:133-42.
20 Van de Werf F, Gore JM, Avezum A, Gulba D, Goodman SG, Budaj A, et al. Access to catheterisation facilities in patients admitted with acute coronary syndromes: multina-

21 Department of Health. The new NHS: modern, dependable. London: Stationery Office, 1997.

(Accepted 24 March 2006)

doi 10.1136/bmj.38849.440914.AE

Northampton General Hospital, Northampton, NN1 5BD John S Birkhead consultant cardiologist

Singleton Hospital, Swansea, Wales

Clive Weston consultant cardiologist

Clinical Effectiveness and Evaluation Unit, Royal College of Physicians of London, London NW1 4LE

Derek Lowe statistician

Correspondence to:J S Birkhead, Clinical Effectiveness and Evaluation Unit, Royal College of Physicians of London, London NW1

4LE John.birkhead@btinternet.com 